\title{
Fabrication of high optical quality Ge-As-Se glasses for the development of low-loss microstructured optical fibers
}

\author{
Marcello Meneghetti ${ }^{\mathrm{a}}$, Céline Caillaud ${ }^{\mathrm{b}}$, Radwan Chahal ${ }^{\mathrm{b}}$, Laurent Brilland ${ }^{\mathrm{b}}$, and Johann \\ Troles $^{\mathrm{a}}$ \\ a Univ Rennes, CNRS, ISCR - UMR 6226 F-35000 Rennes, France \\ ${ }^{\mathrm{b}}$ SelenOptics, 263 Avenue Gal Leclerc, 35042 Rennes, France
}

\begin{abstract}
In the last twenty years the field of chalcogenide glasses has seen increasing interest, due to their broad transparency window in the mid-IR. Furthermore, chalcogenides are showing one of the highest nonlinear refractive indices among glasses. Due to these reasons, the development of chalcogenide microstructured optical fibers with low optical losses can allow for new breakthroughs in various research fields, e.g. new mid-IR laser sources, mid-IR spectroscopy, sensing and applications based on nonlinear effects, like supercontinuum generation. In this framework, chalcogenide glasses with the lowest possible amount of impurities are needed to minimize absorption losses. This study is focused on the attempt of eliminating the pollutants usually giving rise to absorption peaks inside the transparency windows: oxygen, hydrogen, carbon and water. Samples were prepared using a double distillation method: getters that can react with the impurities during the synthesis were added to the initial charge, and the reaction byproducts were eliminated by a two-steps distillation process. $\mathrm{Ge}_{10} \mathrm{As}_{22} \mathrm{Se}_{68}$ was chosen as the system to study because of its nonlinear, optical and thermomechanical properties. Different combinations of chlorides (for the elimination of hydrogen and carbon) and metals (for the elimination of oxygen) were used, and the attenuation spectra of the resulting glasses were compared. The chosen chlorides are $\mathrm{TeCl}_{4}$, $\mathrm{SeCl}_{4}, \mathrm{SbCl}_{3}, \mathrm{GaCl}_{3}$; the metals are $\mathrm{Mg}, \mathrm{Al}, \mathrm{Zr}$, Ni. A holey fiber has been realized by casting method using the best sample, showing that the method is suitable for this composition and that the attenuation before and after the casting are comparable.
\end{abstract}

Keywords: Mid-Infrared, Chemical synthesis methods, Chalcogenide glasses, Microstructured optical fibers

\section{INTRODUCTION}

In the last twenty years the field of chalcogenide glasses has seen increasing interest, thanks to the broad transparency band they exibhit in the mid-infrared, up to wavelengths as high as $20 \mu \mathrm{m}$. This is an extremely interesting region of the light spectrum, and devices able to operate in it can have applications in many fields. In particular, chalcogenide fibers, that can have transmission in the $1-12 \mu \mathrm{m}$ range, ${ }^{1}$ can be especially suitable for telecommunications, sensing, imaging and spectroscopy. ${ }^{2,3}$ Another important property of chalcogenides is showing one of the highest nonlinear refractive indices among glasses. For example, $\mathrm{n}_{2}$ is around two orders of magnitude larger than the one of silica in sulfides and up to 1000 times larger than the one of silica in glasses based on heavier chalcogens (Se, Te). This high nonlinearity permits to observe nonlinear effects even in short fibers. ${ }^{4-7}$ This allows for applications such as slow light (based on stimulated Brillouin scattering), ${ }^{5}$ Raman amplification, ${ }^{8} 3 \mathrm{R}$ regeneration, ${ }^{8,9}$ all-optical switching ${ }^{10}$ and supercontinuum generation. ${ }^{11-13}$ Of particular interest for nonlinear applications are microstructured optical fibers (MOFs), in which guiding properties are mainly defined by the $\mathrm{d} / \Lambda$ ratio (d: diameter of the holes, $\Lambda$ : distance between the holes); ${ }^{14}$ in this kind of fiber the design can be altered to obtain large core single-mode, endlessly single-mode or polarization-maintaining fibers, as well as to enhance nonlinear properties and manage the chromatic dispersion. ${ }^{15}$ Low losses chalcogenide MOFs obtained by using a casting method instead of the more typical stack-and-draw method have been demonstrated. ${ }^{7}$

Further author information: (Send correspondence to M. Meneghetti)

M. Meneghetti: E-mail: marcello.meneghetti@univ-rennes1.fr 
Because of the position of their transparency window, of their high nonlinear refractive index and of their good thermo-mechanical properties, this work focuses on Ge-As-Se ternary glasses, and in particular on the $\mathrm{Ge}_{10} \mathrm{As}_{22} \mathrm{Se}_{68}$ composition, which inside the glass domain of this system has been demonstrated to be highly suitable for the development of small core MOFs thanks to its stability against crystallization. ${ }^{16}$

For all of the abovementioned applications of chalcogenide fibers, it's fundamental to minimize optical losses. A first step toward this result is to obtain as pure as possible parent glasses, in order to reduce impurities-related absorption bands and scattering from particles. In the case of the Ge-As-Se system, the main bands to be reduced, because of their overlapping with the transparency window of our material, are the ones related to the vibrations of $\mathrm{O}-\mathrm{H}, \mathrm{Se}-\mathrm{H}$, and $\mathrm{As}-\mathrm{O}$ bonds. ${ }^{17,18}$ Removal of carbon is also important, because it can form particles that act as scattering centers. In order to remove hydrogen, oxygen, carbon and water, a method similar to the one described ${ }^{19}$ was used: the syntheses have been performed in vacuum atmosphere in a sealed silica crucible, with the addition of getters, i.e. chemical compounds which purpose is to react with oxygen, carbon and hydrogen and give as byproducts either highly refractory or highly volatile compounds. This allows to use distillation to strongly decrease the amount of hydrogen, oxygen and carbon in the glass. The getters used in this physico-chemical process are usually halides for removing hydrogen ${ }^{20}$ and metals for removing oxygen. ${ }^{21}$

While the most commonly used getters are $\mathrm{TeCl}_{4}$ and $\mathrm{Al}$ or $\mathrm{Mg},{ }^{22-24}$ this study aims to introduce some novel ones: $\mathrm{SeCl}_{4}, \mathrm{SbCl}_{3}, \mathrm{GaCl}_{3}$, being chlorides of elements that can be included in the network of Ge-As-Se glasses without negative effects, and $\mathrm{Zr}, \mathrm{Ni}$, which high melting temperature oxides can be removed by distillation. By comparison between these different getters, this work aims at bringing new information on the purification procedure, with the goal to improve the transmission of microstructured optical fibers based on high purity chalcogenide glasses.

\section{EXPERIMENTAL}

We prepared different glasses by melt quenching technique in sealed tube with a double distillation to remove impurities: the charge and getters, placed in a sealed silica tube under high vacuum, were heated up to $800^{\circ} \mathrm{C}$ and subsequently quenched in water; after this step, they were annealed at a temperature just below $\mathrm{T}_{\mathrm{g}}$ in order to reduce the internal stress. The charge was prepared with pure elements, nominally germanium $5 \mathrm{~N}$, arsenic $5 \mathrm{~N}$ and selenium $5 \mathrm{~N}$, weighted and inserted in the tube in a glovebox, under inert atmosphere. During the melting of the charge, the reaction of the getters with the impurities occurs: the halides react with hydrogen and carbon, following reactions like the ones presented as an example below (only the initial and final states are shown, intermediate reaction steps are not well understood)

$$
\begin{gathered}
4 \mathrm{Se}-\mathrm{H}+\mathrm{TeCl}_{4} \rightleftharpoons 4 \mathrm{Se}+\mathrm{Te}+4 \mathrm{HCl}{ }^{22} \\
\mathrm{C}+\mathrm{TeCl}_{4} \rightleftharpoons \mathrm{Te}+\mathrm{CCl}_{4}
\end{gathered}
$$

while the metals react with the oxygen in a way similar to the one shown below for the case of magnesium.

$$
\mathrm{As} \longrightarrow \mathrm{O}+\mathrm{Mg} \rightleftharpoons \mathrm{As}+\mathrm{MgO}
$$

The resulting glass underwent a two step distillation, with temperature and duration of the steps in this procedure kept fixed along the different samples prepared for the comparison. The first one happens in a open setup, in connection with a pump actively removing gases (dynamic distillation). The second one is performed in a sealed setup, once again in high vacuum atmosphere (static distillation). A schematic of these processes can be found in Fig. 1. During the dynamic distillation the glass migrates from the hot zone, at a temperature of $600^{\circ} \mathrm{C}$, to the cold zone. Metal oxides and residuals of silica from the crucible, being refractory, do not migrate, while water and hydrohalic acids, being volatile, are sucked into the liquid nitrogen cooled trap by the vacuum pump, and condensate there. The second step removes traces of metal oxides that could have remained inside the glass. After the distillations, the glass was melted, quenched and annealed again with the same procedure used for the first synthesis. This last step is fundamental to homogenize the material, which during the distillations was separated into compounds with different melting temperatures.

All the silica setups used were made of low $\mathrm{OH}$ content silica $(<3 \mathrm{ppm})$, and were etched with hydrofluoric acid before being used, in order to minimize the addition of further impurities to the glass, especially after the 
distillations. The glass composition before and after the distillation process was checked with energy dispersive spectroscopy (EDS) measurements. The variations in the stoichiometry, when measurable, were below $1 \%$, and no leftover traces of getters were found in the glass after the distillation.

As for the different getters used in this comparison, $\mathrm{TeCl}_{4}, \mathrm{SeCl}_{4}, \mathrm{SbCl}_{3}, \mathrm{GaCl}_{3}$ were chosen as halides, while as metals $\mathrm{Mg}, \mathrm{Al}, \mathrm{Zr}$ and $\mathrm{Ni}$ were used. The ratio between the mass of both getters (one halide and one metal for each sample) and the total mass of glass is kept fixed along all the samples. Specifically, each sample contains 2000 ppmw of hydrogen getter and 200 ppmw of oxygen getter. It is to be noticed that hydrogen getters used are all chlorides of elements well compatible with the chalcogenide glass network. Thanks to this and to the low concentration, those elements should not affect the optical properties in case they entered the glass during distillation.

In order to see the effect of the purification, the so-obtained glasses were then drawn in $125 \mu \mathrm{m}$ fibers, the attenuation of which was measured by cut-back technique on a Bruker Tensor 37 FTIR spectrometer using an external liquid nitrogen cooled MCT detector. For each measurement two cut-backs were performed: one with a long part of fiber for the measurement of the low (background) attenuation and one with a short length of fiber for the measurement of the high (peaks) attenuation. The results of the two measurements were then combined into complete spectra. The error was estimated by comparison of repeated measurements on the same fiber, and like the experiment can be separated in two regimes: an error of about $0.1 \mathrm{~dB} / \mathrm{m}$ for the background, where detection-related sources of error are dominant, and an error of about $5 \%$ for the peaks, where because of the short length of fiber random errors related to the cleaving of the fiber are dominant.

For each combination of getter studied, several syntheses were performed. Only the best results achieved will be shown in this study.

Holey preforms were then obtained by casting method $^{7}$ using some of the most promising glasses, and drawn into microstructured endlessly single mode fibers, which were characterized using once again FTIR spectrometry.

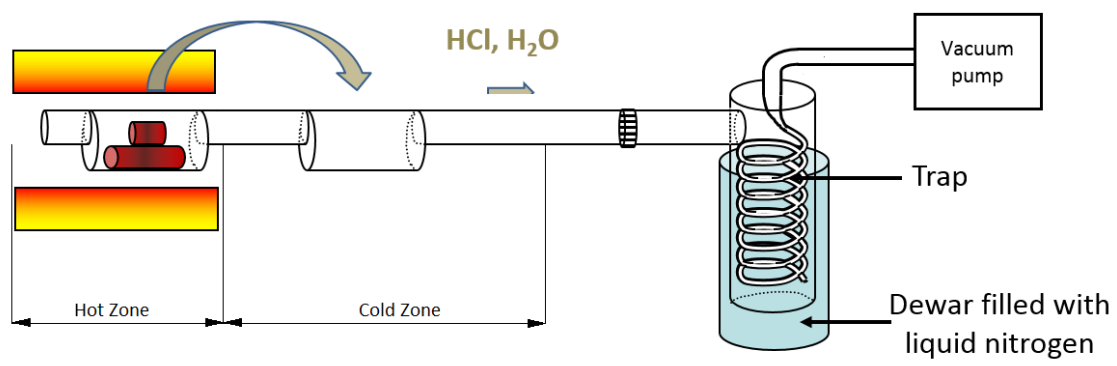

a)

b)

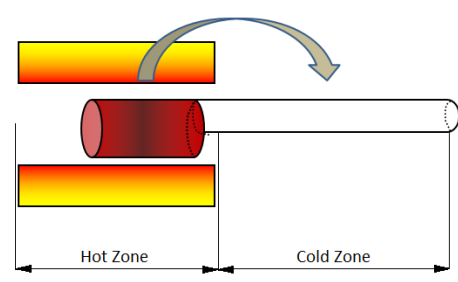

Figure 1: Schematics of the dynamic (a) and static (b) distillation processes.

\section{RESULTS AND DISCUSSION}

In order to compare the effect of different hydrogen getters on the Se-H absorption peak $(4.5 \mu \mathrm{m})$, a series of sample was prepared fixing $\mathrm{Mg}$ as the oxygen getter in combination with the different halides listed in the "EXPERIMENTAL" Section. In a similar way, a second series was prepared using $\mathrm{TeCl}_{4}$ as an hydrogen getter and the metals listed in the "EXPERIMENTAL" Section, with the intention of studying the Ge-O and As-O peaks $\left(7.9 \mu \mathrm{m}\right.$ and $8.9 \mu \mathrm{m}$ respectively). $\mathrm{Mg}$ and $\mathrm{TeCl}_{4}$ were chosen as fixed getters for the comparison because 
their use has already been documented on this composition. ${ }^{16}$ The measured losses in the transparency windows for the two series are visible in Fig. 2, together with an unpurified glass to show the huge improvements in terms of transmission given by the purification.

The absence of visible carbon-related bands in any of the purified samples can also be noticed. Indeed, in our case carbon impurities are mainly particles, and they therefore induce scattering losses rather than absorption ones.
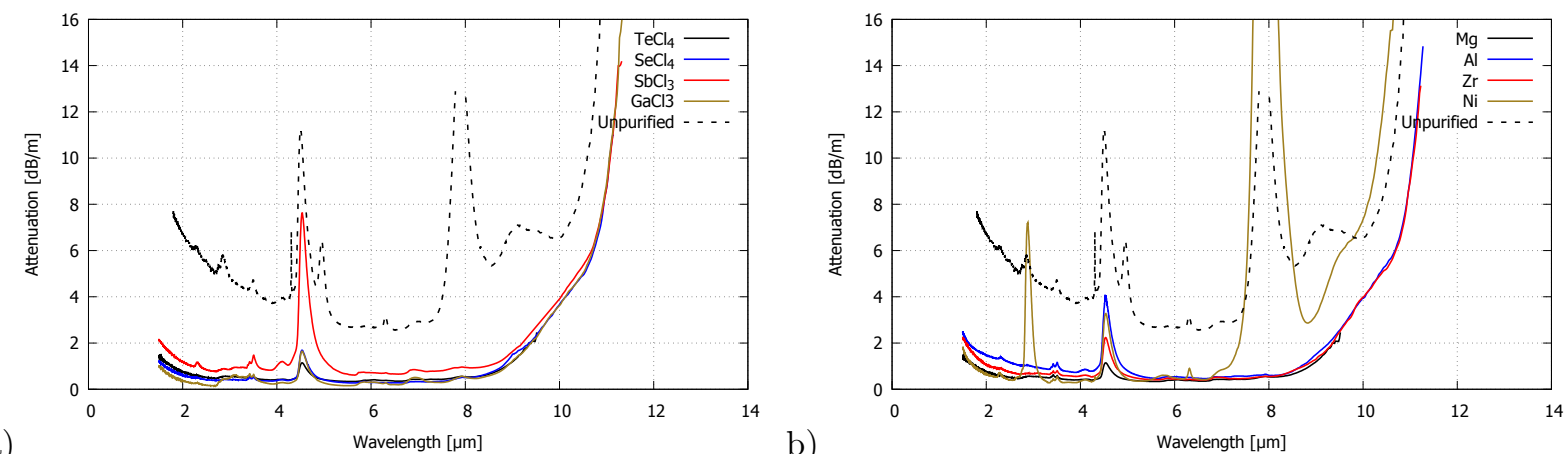

a) Wavelength [um]

b)

Figure 2: Comparison between the attenuation spectra of $\mathrm{Ge}-\mathrm{As}$ - Se fibers prepared using magnesium in combination with different hydrogen getters (a) and $\mathrm{TeCl}_{4}$ in combination with different oxygen getters (b). In both graphs, an example of non purified glass is shown.

As it can be seen in Fig. 2.a, $\mathrm{TeCl}_{4}, \mathrm{SeCl}_{4}$ and $\mathrm{GaCl}_{3}$, reducing the intensity of the $\mathrm{Se}-\mathrm{H}$ peak to a level of around $1.5 \mathrm{~dB} / \mathrm{m}$, seem to be considerably more efficient than $\mathrm{SbCl}_{3}$. In order to better understand the reason of this difference, a starting point could be comparing the actual amount of chlorine ions introduced in the melt by the addition of the different halides, for which the mass was kept constant, under the assumption of full dissociation of all the compounds.

Table 1: Molar content of chlorine introduced in the melt by different getters.

\begin{tabular}{|r|c|}
\hline Halide & Cl amount $\left(\mathrm{mol} \cdot 10^{-3}\right)$ \\
\hline $\mathrm{TeCl}_{4}$ & $1.75 \pm 0.15$ \\
\hline $\mathrm{SeCl}_{4}$ & $2.1 \pm 0.2$ \\
\hline $\mathrm{SbCl}_{3}$ & $1.6 \pm 0.1$ \\
\hline $\mathrm{GaCl}_{3}$ & $2.0 \pm 0.2$ \\
\hline
\end{tabular}

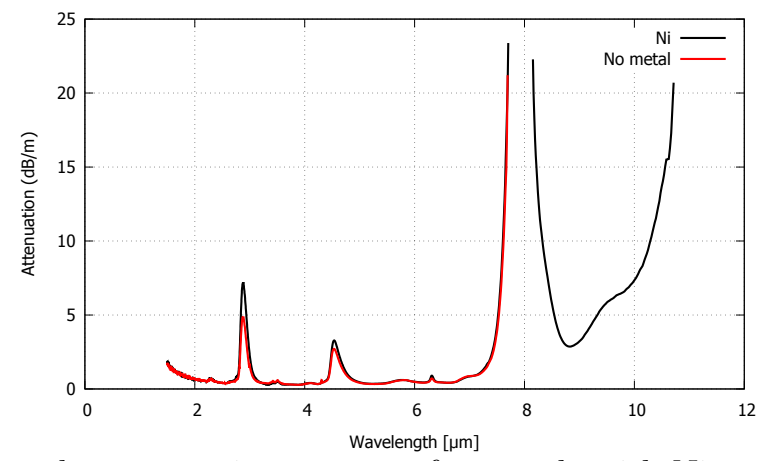

Figure 3: Comparison between the attenuation spectra of a sample with $\mathrm{Ni}$ as a metal and a sample with no metal at all. In both samples $\mathrm{TeCl}_{4}$ is used as hydrogen getter. 
No relation between the theoretical moles of $\mathrm{Cl}$ and the efficiency appears to emerge from the values presented in Table 1, with $\mathrm{TeCl}_{4}$ resulting to be the most efficient getter despite the relatively low total amount of chlorine it can introduce in the system. In accordance with the absence of this relation, increasing the mass of the getters didn't bring to better results. Also, comparing the standard Gibbs free energies of the different getters and of $\mathrm{HCl}$ in the range of temperatures ${ }^{25-27}$ used during the synthesis, we see no trivial correlation between the stability of these chlorides and their efficiency; on the contrary, in a counterintuitive way the most stable compound, nominally $\mathrm{GaCl}_{3}$ gives better results than the less stable $\mathrm{SbCl}_{3}$. This could be due to different elements in the melt acting as catalysts to favor the reaction in a way similar to the one suggested by Nguyen et.al. ${ }^{28}$ the individuation of the actual reaction steps is far from the goals of this paper, but the fact that the partial elimination of $\mathrm{Se}-\mathrm{H}$ bonds happens even without the addition of any metal to the melt (Fig. 3), a cooperative action of the two getters seems less probable with respect to the action of other glass or crucible components as catalysts.

From Fig. 2.b, it can be clearly seen that oxygen-wise magnesium, aluminum and zirconium efficiently suppress the Ge-O and As-O bands in the attenuation spectra of the respective samples. Nickel, on the other side, seems to be completely ineffective in this regard: as visible in Fig. 3, the spectrum of the Ni containing sample is almost perfectly superimposable with the one of a sample prepared with the same amount of $\mathrm{TeCl}_{4}$, but no oxygen getter. This is compatible with the Ellingham diagrams shown in Fig. 4.a, from which formation of $\mathrm{NiO}$ seem less favored than formation of $\mathrm{GeO}_{2}$, whereas formation of $\mathrm{MgO}, \mathrm{Al}_{2} \mathrm{O}_{3}$ and $\mathrm{ZrO}_{2}$ appear to be strongly favored against Germanium dioxide. The peaks at $2.9 \mu \mathrm{m}$ and $6.3 \mu \mathrm{m}$ appearing in the non-purified and Ni-containing samples are assigned to $\mathrm{O}-\mathrm{H}$ and $\mathrm{H}_{2} \mathrm{O}$ respectively.

While the choice of the halide doesn't seem here to influence the content of oxides in a relevant way, when comparing the Se-H peaks of the samples prepared with different oxygen getters there is a significant difference. In this case too, the explanation from a reaction dynamics point of view is really complicated and would require deeper studies. Anyway, given the high absolute value of the formation $\Delta \mathrm{G}$ of ternary compounds in the form $\mathrm{M}_{\mathrm{x}} \mathrm{Si}_{\mathrm{y}} \mathrm{O}_{\mathrm{z}}$, with $\mathrm{M}$ being a metal, shown in Fig. 4.b for $\mathrm{Al}$, $\mathrm{Zr}$ and $\mathrm{Mg}$, it is reasonable to suppose that the oxygen getters can interact with the silica crucible. This could cause the release of hydrogen from $\mathrm{Si}-\mathrm{O}-\mathrm{H}$ groups at the surface of the crucible, therefore influencing the amount of hydrogen-related impurities in the chalcogenide glass.
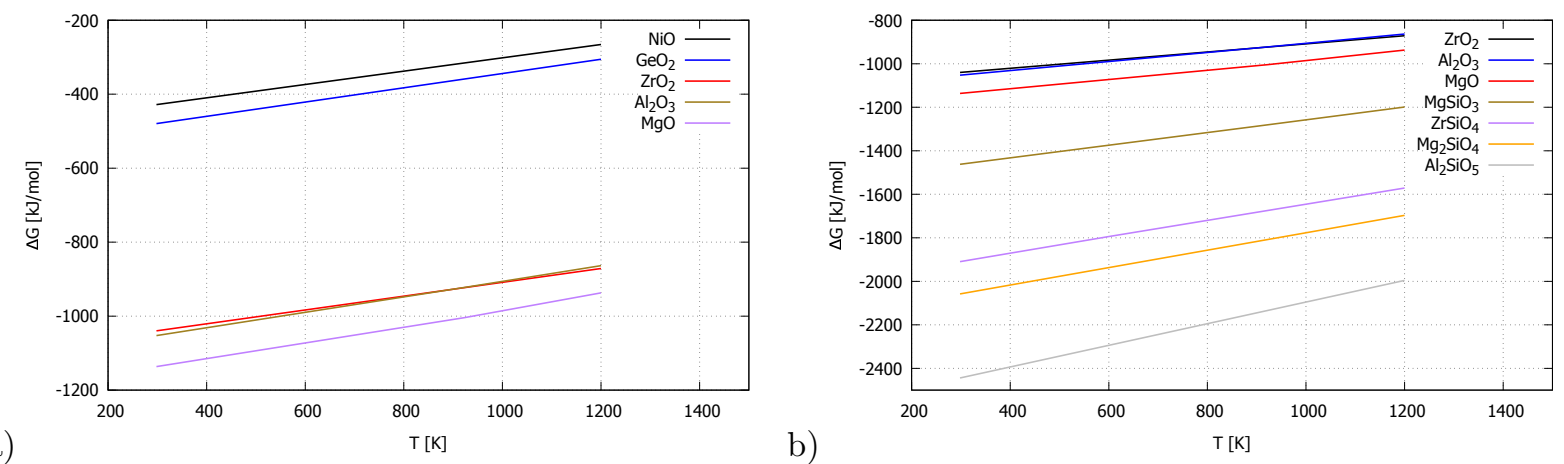

Figure 4: Ellingham diagrams of different oxygen compounds. ${ }^{25,26}$

For all of these reasons, it appears clearly that the choice of the getters, as well as the amount of getters used for the reactions, used has to be the result of a compromise between the reduction of the bands related to hydrogen and oxygen pollutions and the necessity to avoid as much as possible reactions involving the crucible. ${ }^{29-31}$ The combination of $\mathrm{TeCl}_{4}$ and magnesium, which has shown the best results in terms of attenuation, has been chosen as the most suitable for the realization by casting $\operatorname{method}^{7}$ of a microstructured fiber, of which attenuation spectrum and cross section are visible in Fig. 5. This fiber has an diameter of $125 \mu \mathrm{m}$ and a core diameter of $11 \mu \mathrm{m}$. The holes diameter (d) is $3.4 \mu \mathrm{m}$, while the pitch $(\Lambda)$ is $7.2 \mu \mathrm{m}$, leading to a $\frac{\mathrm{d}}{\Lambda}$ parameter of 0.47 . As visible from Fig. 5.a, the process of casting has a small influence on both the background and the impurities, with just a small increasing of the $\mathrm{Se}-\mathrm{H}$ band and the appearance of small peaks related to water and oxygen pollution. In addition, it has been shown that an optimized purification of the chalcogenide glass allows to mold 

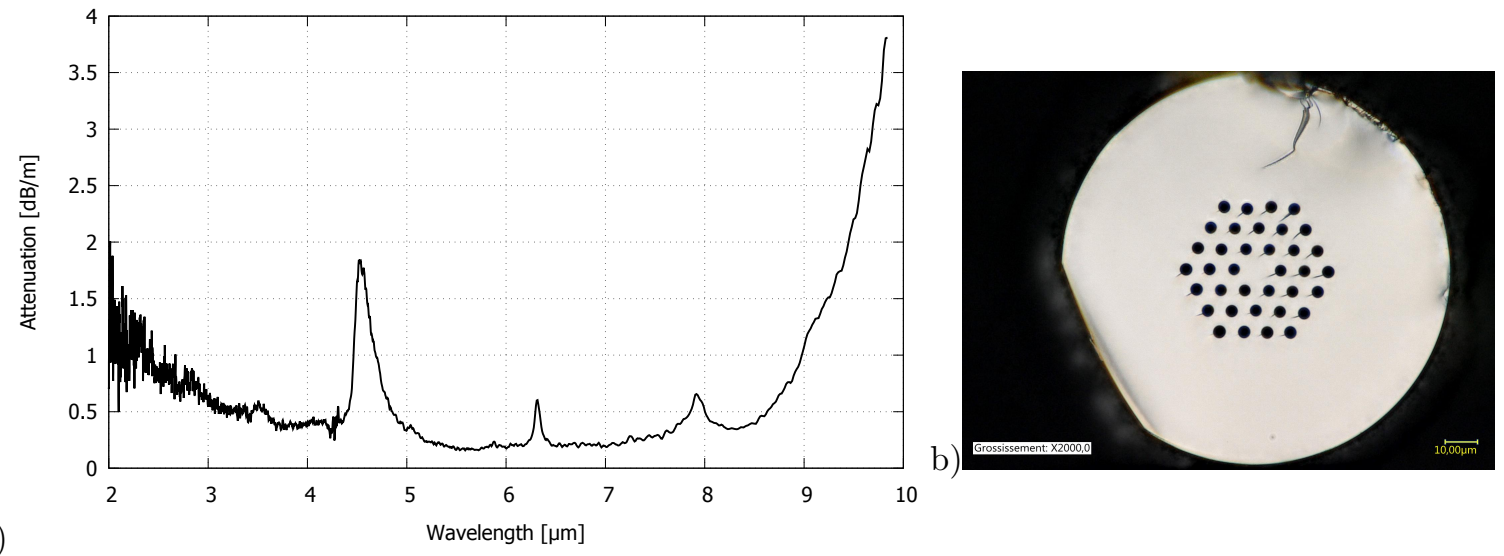

a)

Figure 5: Attenuation spectrum (a) and cross section (b) of a holey fiber obtained from the sample containing

$\mathrm{Mg}$ and $\mathrm{TeCl}_{4}$.

and draw chalcogenide holey fibers with optical losses $<0.2 \mathrm{~dB} / \mathrm{m}$ (at $5.5 \mu \mathrm{m}$ ) and Se-H absorption losses lower than $2 \mathrm{~dB} / \mathrm{m}$. This is the best transmission ever obtained in a selenide holey fiber.

\section{CONCLUSIONS}

The presented results show that it is possible to obtain high purity $\mathrm{Ge}_{10} \mathrm{As}_{22} \mathrm{Se}_{68}$ by double distillation method, and that the efficacy of this method is heavily dependent on the choice of the getters. Between the ones tested, $\mathrm{TeCl}_{4}$ and $\mathrm{Mg}$ seem to be the best candidates to minimize the incidence of both oxygen and hydrogen pollution on the optical losses of fibers.

When choosing the species and amounts of halides and metals to introduce in the system, given that appearently they can react with the silica crucible, decreasing the final quality of the glass.

Further research will be needed in the future on this purification method, mainly focusing on the influence of the weight ratios of getters to be introduced in the glass.

The viability of casting method for obtaining high quality endlessly single mode fibers, with an attenuation lower than $0.2 \mathrm{~dB} / \mathrm{m}$ at $5.5 \mu \mathrm{m}$, using this composition and a proper purification route is also demonstrated. This is of particular interest, seeing how chalcogenide microstructured optical fibers are an enabling technology for many applications in different fields.

\section{ACKNOWLEDGMENTS}

This project has received funding from the European Union's Horizon 2020 research and innovation program under Grant Agreement No. 722380.

\section{REFERENCES}

[1] J. Troles, V. Shiryaev, M. Churbanov, P. Houizot, L. Brilland, F. Désévédavy, F. Charpentier, T. Pain, G. Snopatin, and J.-L. Adam, "Gese4 glass fibres with low optical losses in the mid-ir," Optical Materials 32(1), pp. 212-215, 2009.

[2] J. Sanghera and I. Aggarwal, "Active and passive chalcogenide glass optical fibers for ir applications: a review," Journal of Non-Crystalline Solids 256, pp. 6-16, 1999.

[3] J. Keirsse, C. Boussard-Plédel, O. Loreal, O. Sire, B. Bureau, B. Turlin, P. Leroyer, and J. Lucas, "Chalcogenide glass fibers used as biosensors," Journal of non-crystalline solids 326, pp. 430-433, 2003.

[4] J. M. Harbold, F. O. Ilday, F. W. Wise, and B. G. Aitken, "Highly nonlinear ge-as-se and ge-as-s-se glasses for all-optical switching," IEEE Photonics Technology Letters 14(6), pp. 822-824, 2002. 
[5] J. S. Sanghera, L. B. Shaw, P. Pureza, V. Q. Nguyen, D. Gibson, L. Busse, I. D. Aggarwal, C. M. Florea, and F. H. Kung, "Nonlinear properties of chalcogenide glass fibers," International Journal of Applied Glass Science 1(3), pp. 296-308, 2010.

[6] M. Asobe, "Nonlinear optical properties of chalcogenide glass fibers and their application to all-optical switching," Optical Fiber Technology 3(2), pp. 142-148, 1997.

[7] Q. Coulombier, L. Brilland, P. Houizot, T. Chartier, T. N. N'guyen, F. Smektala, G. Renversez, A. Monteville, D. Méchin, T. Pain, et al., "Casting method for producing low-loss chalcogenide microstructured optical fibers," Optics Express 18(9), pp. 9107-9112, 2010.

[8] R. E. Slusher, G. Lenz, J. Hodelin, J. Sanghera, L. B. Shaw, and I. D. Aggarwal, "Large raman gain and nonlinear phase shifts in high-purity as 2 se 3 chalcogenide fibers," JOSA B 21(6), pp. 1146-1155, 2004.

[9] L. Fu, M. Rochette, V. Ta'eed, D. Moss, and B. Eggleton, "Investigation of self-phase modulation based optical regeneration in single mode as 2 se 3 chalcogenide glass fiber," Optics Express 13(19), pp. 7637-7644, 2005.

[10] M. Asobe, T. Kanamori, and K. Kubodera, "Ultrafast all-optical switching using highly nonlinear chalcogenide glass fiber," IEEE photonics technology letters 4(4), pp. 362-365, 1992.

[11] U. Møller, Y. Yu, I. Kubat, C. R. Petersen, X. Gai, L. Brilland, D. Méchin, C. Caillaud, J. Troles, B. LutherDavies, et al., "Multi-milliwatt mid-infrared supercontinuum generation in a suspended core chalcogenide fiber," Optics express 23(3), pp. 3282-3291, 2015.

[12] C. R. Petersen, U. Møller, I. Kubat, B. Zhou, S. Dupont, J. Ramsay, T. Benson, S. Sujecki, N. AbdelMoneim, Z. Tang, et al., "Mid-infrared supercontinuum covering the 1.4-13.3 $\mu \mathrm{m}$ molecular fingerprint region using ultra-high na chalcogenide step-index fibre," Nature Photonics 8(11), p. 830, 2014.

[13] C. R. Petersen, R. D. Engelsholm, C. Markos, L. Brilland, C. Caillaud, J. Trolès, and O. Bang, "Increased mid-infrared supercontinuum bandwidth and average power by tapering large-mode-area chalcogenide photonic crystal fibers," Optics express 25(13), pp. 15336-15348, 2017.

[14] J. Knight, T. Birks, P. S. J. Russell, and D. Atkin, "All-silica single-mode optical fiber with photonic crystal cladding," Optics letters 21(19), pp. 1547-1549, 1996.

[15] G. Renversez, B. Kuhlmey, and R. McPhedran, "Dispersion management with microstructured optical fibers: ultraflattened chromatic dispersion with low losses," Optics Letters 28(12), pp. 989-991, 2003.

[16] P. Toupin, L. Brilland, J. Trolès, and J.-L. Adam, "Small core ge-as-se microstructured optical fiber with single-mode propagation and low optical losses," Optical Materials Express 2(10), pp. 1359-1366, 2012.

[17] J. Ballato, H. Ebendorff-Heidepriem, J. Zhao, L. Petit, and J. Troles, "Glass and process development for the next generation of optical fibers: A review," Fibers 5(1), p. 11, 2017.

[18] M. Churbanov, "High-purity chalcogenide glasses as materials for fiber optics," Journal of non-crystalline solids 184, pp. 25-29, 1995.

[19] V. Shiryaev, M. Churbanov, E. Dianov, V. Plotnichenko, J. Adam, and J. Lucas, "Recent progress in preparation of chalcogenide as-se-te glasses with low impurity content," Journal of optoelectronics and advanced materials $\mathbf{7}(4)$, p. 1773, 2005.

[20] D. Lezal, J. Pedlikova, J. Gurovic, and R. Vogt, "The preparation of chalcogenide glasses in chlorine reactive atmosphere," Ceramics 40(2), pp. 55-59, 1996.

[21] 张斌, 杨志勇, 任和, 张鸣杰, 章健, and 唐定远, "Preparation method of high-purity and low-loss chalcogenide glass," 17 2015. CN103332851B.

[22] V. Q. Nguyen, J. S. Sanghera, B. Cole, P. Pureza, F. H. Kung, and I. D. Aggarwal, "Fabrication of arsenic sulfide optical fiber with low hydrogen impurities," Journal of the American Ceramic Society 85(8), pp. 2056-2058, 2002.

[23] S. Danto, D. Thompson, P. Wachtel, J. D. Musgraves, K. Richardson, and B. Giroire, "A comparative study of purification routes for as2se3 chalcogenide glass," International Journal of Applied Glass Science 4(1), pp. 31-41, 2013.

[24] Z. Tang, V. S. Shiryaev, D. Furniss, L. Sojka, S. Sujecki, T. M. Benson, A. B. Seddon, and M. F. Churbanov, "Low loss ge-as-se chalcogenide glass fiber, fabricated using extruded preform, for mid-infrared photonics," Optical Materials Express 5(8), pp. 1722-1737, 2015.

[25] T. B. Reed, Free energy of formation of binary compounds, MIT press, 1971. 
[26] W. Malcolm Jr, NIST-JANAF thermochemical tables, American Institute of Physics; The American Chemical Society, 1998.

[27] W. M. Haynes, CRC handbook of chemistry and physics, CRC press, 2014.

[28] V. Q. Nguyen, G. Drake, G. Villalobos, D. Gibson, S. Bayya, W. Kim, C. Baker, G. Chin, F. H. Kung, M. I. Kotov, et al., "Effect of aluminum and tellurium tetrachloride addition on the loss of arsenic selenide optical fiber," Optical Materials 64, pp. 327-333, 2017.

[29] V. Shiryaev, S. Mishinov, and M. Churbanov, "Investigation of adhesion of chalcogenide glasses to silica glass," Journal of Non-Crystalline Solids 408, pp. 71-75, 2015.

[30] S. Mishinov, M. Churbanov, A. Gorokhov, D. Kazakov, V. Shiryaev, A. Suchkov, L. Igumnov, and G. Snopatin, "Adhesion mechanism of destruction of silica-glass surface during the preparation and treatment of optical glassy arsenic chalcogenides," Inorganic Materials 52(7), pp. 716-720, 2016.

[31] M. Churbanov, S. Mishinov, V. Shiryaev, and L. Ketkova, "Contamination of glassy arsenic sulfide by sio2 particles during melt solidification in silica glassware," Journal of Non-Crystalline Solids 480, pp. 3-7, 2018. 